WIS-99/31/Sept-DPP

SLAC-PUB-8244

hep-ph/9909390

September 1999

\title{
Can lepton flavor violating interactions explain the atmospheric neutrino problem?
}

\author{
Sven Bergmann, ${ }^{a}$ Yuval Grossman ${ }^{b}$ and Damien M. Pierce ${ }^{c}$ \\ ${ }^{a}$ Department of Particle Physics \\ Weizmann Institute of Science, Rehovot 76100, Israel \\ ${ }^{b}$ Stanford Linear Accelerator Center \\ Stanford University, Stanford, CA 94309 \\ ${ }^{c}$ Brookhaven National Laboratory \\ Upton, NY 11973
}

\begin{abstract}
We investigate whether flavor changing neutrino interactions (FCNIs) can be sufficiently large to provide a viable solution to the atmospheric neutrino problem. Effective operators induced by heavy boson exchange that allow for flavor changing neutrino scattering off quarks or electrons are related by an $S U(2)_{L}$ rotation to operators that induce anomalous tau decays. Since $S U(2)_{L}$ violation is small for New Physics at or above the weak scale, one can use the upper bounds on lepton flavor violating tau decays or on lepton universality violation to put severe, model-independent bounds on the relevant non-standard neutrino interactions. Also Z-induced flavor changing neutral currents, due to heavy singlet neutrinos, are too small to be relevant for the atmospheric neutrino anomaly. We conclude that the FCNI solution to the atmospheric neutrino problem is ruled out.
\end{abstract}

Research at SLAC is supported by the U.S. Department of Energy under contract DE-AC03-76SF00515. 


\section{INTRODUCTION}

Several atmospheric neutrino (AN) experiments [1] observe an anomalous ratio $\nu_{e} / \nu_{\mu}$ in the atmospheric neutrino flux. This long-standing AN problem has been confirmed by the recent Super-Kamiokande high-statistics observations [i-1], which give strong evidence that the standard model (SM) description of the neutrino sector is incomplete. The standard solution to the AN anomaly in terms of neutrino oscillations requires that neutrinos are massive, and that there is mixing in the lepton sector. Then, $\nu_{\mu} \rightarrow \nu_{\tau}$ oscillations can explain the atmospheric neutrino data provided that the relevant mass-squared difference is $\Delta_{A N} \sim 10^{-3} \mathrm{eV}^{2}$ and the muon and tau neutrinos have large vacuum mixing angles, $\sin 2 \theta_{A N} \sim 1$.

Recently, an alternative solution, where the AN anomaly is induced by non-standard neutrino interactions has been proposed [ [n to be massless, but they are subject to non-standard interactions. For neutrino propagation in matter, flavor changing neutrino interactions (FCNIs) induce an off-diagonal term in the effective neutrino mass matrix, while non-universal flavor diagonal interactions generate the required splitting between the diagonal terms. A priori such a scenario is well motivated, since many extension of the SM predict new neutrino interactions. Moreover, it is well known

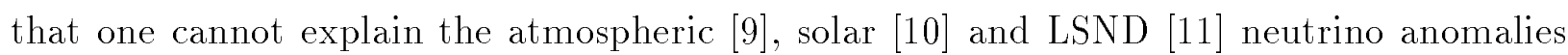
with three light neutrinos. Thus, rather than ignoring one of the results or introducing a forth, light sterile-neutrino [i] $\left.{ }_{1}\right]$, it is interesting to investigate whether FCNIs can explain

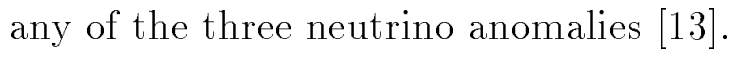

The two effective parameters that describe the non-standard interactions of $\nu_{\mu}$ and $\nu_{\tau}$

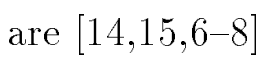

$$
\epsilon_{\nu}^{f} \equiv \frac{G_{\nu_{\mu} \nu_{\tau}}^{f}}{G_{F}} \quad \text { and } \quad \epsilon_{\nu}^{\prime f} \equiv \frac{G_{\nu_{\tau} \nu_{\tau}}^{f}-G_{\nu_{\mu} \nu_{\mu}}^{f}}{G_{F}}
$$

where $G_{\nu_{\alpha} \nu_{\beta}}^{f}(\alpha, \beta=\mu, \tau$ and $f=u, d, e)$ denotes the effective coupling of the four fermion operator

$$
\mathcal{O}_{\nu}^{f} \equiv\left(\overline{\nu_{\alpha}} \nu_{\beta}\right)(\bar{f} f)
$$

The Lorentz structure of $\mathcal{O}_{\nu}^{f}$ depends on the New Physics that induces this operator. Operators which involve only left-handed neutrinos (and which conserve total lepton number) can be decomposed into a $(V-A) \otimes(V-A)$ and a $(V-A) \otimes(V+A)$ component. (Any single New Physics contribution that is induced by chiral interactions yields only one of these two components.) It is, however, important to note that only the vector part of the background fermion current affects the neutrino propagation for an unpolarized medium at rest $\left[\underline{1}_{\overline{1}}^{\overline{1}} \overline{\hat{v}_{1}}\right]$. Hence only the $(V-A) \otimes(V)$ part of $\mathcal{O}_{\nu}^{f}$ is relevant for neutrino oscillations in normal matter. One mechanism to induce such operators is due to the exchange of heavy bosons that appear in various extensions of the standard model. An alternative mechanism 
arises when extending the fermionic sector of the standard model and is due to $Z$-induced

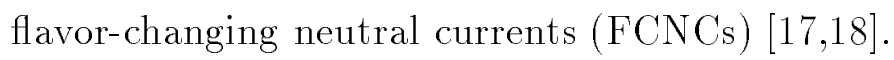

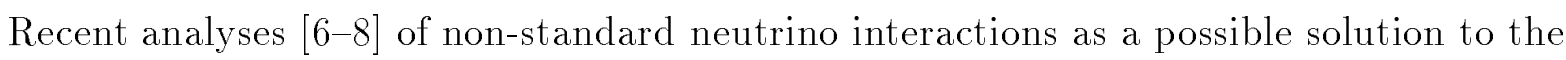
atmospheric neutrino data suggest that FCNIs can provide a good fit to the data provided that

$$
\epsilon_{\nu}^{q}, \epsilon_{\nu}^{\prime q} \gtrsim 0.1 \text { or } \epsilon_{\nu}^{e}, \epsilon_{\nu}^{\prime e} \gtrsim 0.3
$$

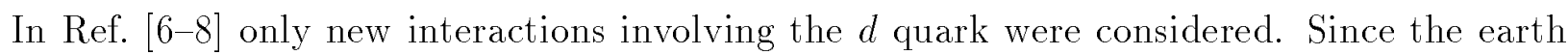
is electrically neutral and its neutron to proton ratio is close to unity, we conclude that the required values for $\epsilon_{\nu}^{q}$ and $\epsilon_{\nu}^{\prime q}$ are similar for $q=d$, $u$, while those for $\epsilon_{\nu}^{e}$ and $\epsilon_{\nu}^{\prime e}$ are larger by a factor of three.

The authors of Ref. $[1,900$ argue that such a scenario does not lead to a good description of the data. In this paper we do not try to resolve this issue, but investigate whether the lower bounds on $\epsilon_{\nu}^{f}$ and $\epsilon_{\nu}^{\prime f}$ in $\left({ }_{1}^{1}-\bar{z}^{\prime}\right)$ are at all phenomenologically viable. The authors of Ref. [8] have estimated the upper bound $\epsilon_{\nu}^{d} \lesssim 0.1-1.0$ from the low energy $\nu_{\mu}$ neutral current cross-section, concluding that FCNIs could be relevant for the AN anomaly. In

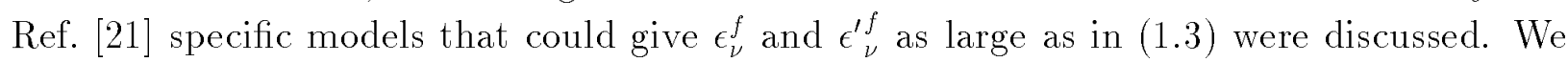
argue, however, that model-independently the upper bounds from related, charged lepton decay data imply that $\epsilon_{\nu}^{f}$ or $\epsilon_{\nu}^{\prime f}$ can be at most at the one-percent level. Thus we conclude that FCNIs do not play a significant role for the atmospheric neutrino problem.

In Section 'ÎI' we investigate in a model-independent framework the constraints on FCNIs that are induced by heavy boson exchange. In most cases the upper bounds on lepton flavor violating tau decays, in particular $\tau^{-} \rightarrow \mu^{-} M\left(M=\pi^{0}, \rho^{0}, \eta\right)$ and $\tau^{-} \rightarrow \mu^{-} e^{+} e^{-}$, imply stringent constraints on $\epsilon_{\nu}^{f}$ that are inconsistent with (1, $\left.{ }_{3}^{\prime}\right)$. In the remaining cases severe constraints on $\epsilon_{\nu}^{\prime f}$ are derived using bounds on lepton universality violation. In Section III I I I we show that also $Z$-induced FCNCs, that arise due to heavy singlet neutrinos, cannot be large enough to explain the AN anomaly. We conclude in Section IV.:

\section{FLAVOR CHANGING NEUTRINO INTERACTIONS INDUCED BY HEAVY BOSON EXCHANGE}

\section{A. Formalism}

The analysis of FCNIs that could be relevant for the AN problem is similar to the

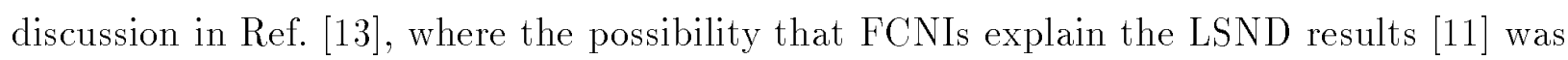
ruled out. In general, the presence of a heavy boson $\mathcal{B}$ that couples to fermion bilinears $B_{i j}$ with the trilinear couplings $\lambda_{i j}$, where $i, j=1,2,3$ refer to fermion generations, gives rise to the four fermion operator $B_{i j}^{\dagger} B_{k l}$ with the effective coupling

$$
G_{N}^{B^{\dagger} B}=\frac{\lambda_{i j}^{*} \lambda_{k l}}{4 \sqrt{2} M_{\mathcal{B}}^{2}},
$$


at energies well below the boson mass $M_{\mathcal{B}}$. Thus, in terms of the trilinear coupling $\lambda_{\alpha f}$ that describes the coupling of some heavy boson $\mathcal{B}$ to $\nu_{\alpha}(\alpha=\mu, \tau)$ and a charged fermion $f=u, d, e$ the effective parameters in (1). $\left.\overline{1}_{1}^{\prime}\right)$ are given by

$$
\epsilon_{\nu}^{f}=\frac{\lambda_{\tau f}^{*} \lambda_{\mu f}}{4 \sqrt{2} M_{\mathcal{B}}^{2} G_{F}} \quad \text { and } \quad \epsilon_{\nu}^{\prime f}=\frac{\left|\lambda_{\tau f}\right|^{2}-\left|\lambda_{\mu f}\right|^{2}}{4 \sqrt{2} M_{\mathcal{B}}^{2} G_{F}} .
$$

The crucial point of our analysis is the following: Since the SM neutrinos are components of $S U(2)_{L}$ doublets, the same trilinear couplings $\lambda_{\alpha f}$ that give rise to non-zero $\epsilon_{\nu}^{f}$ or $\epsilon_{\nu}^{\prime f}$ also induce other four-fermion operators. These operators involve the $S U(2)_{L}$ partners of the neutrinos, i.e. the charged leptons, and can be used to constrain the relevant couplings.

\begin{tabular}{|c||c||c||c|}
\hline Bilinear $B$ & Couples to Boson $\mathcal{B}$ & Example & $\left(M_{1} / M_{2}\right)_{\max }^{2}$ \\
\hline \hline$(L L)_{s}$ & $\mathcal{S}(\mathbf{1}, \mathbf{1}, 1)$ & $\tilde{\ell}_{R}^{c}\left(\mathrm{SUSY} \not R_{p}\right)$ & \\
\hline $\bar{L} \ell_{R}$ & $\mathcal{S}(\mathbf{1}, \mathbf{2}, 1 / 2)$ & $\tilde{L}^{c}\left(\mathrm{SUSY} \not \boldsymbol{R}_{p}\right)$ & 6.8 \\
\hline$(L L)_{t}$ & $\mathcal{S}(\mathbf{1}, \mathbf{3}, 1)$ & $\Delta_{L}(\mathrm{LRSM})$ & 5.9 \\
\hline \hline$(\bar{L} L)_{s}$ & $\mathcal{V}(\mathbf{1}, \mathbf{1}, 0)$ & & \\
\hline$L \ell_{R}$ & $\mathcal{V}(\mathbf{1}, \mathbf{2}, 3 / 2)$ & & \\
\hline$(\bar{L} L)_{t}$ & $\mathcal{V}(\mathbf{1}, \mathbf{3}, 0)$ & & \\
\hline \hline$\overline{e_{R}} e_{R}$ & $\mathcal{V}(\mathbf{1}, \mathbf{1}, 0)$ & & \\
\hline
\end{tabular}

Tab. 1: Lepton-Lepton Bilinears

\begin{tabular}{|c||c||c||c|}
\hline Bilinear $B$ & Couples to Boson $\mathcal{B}$ & Example & $\left(M_{1} / M_{2}\right)_{\max }^{2}$ \\
\hline \hline$(L Q)_{s}$ & $\mathcal{S}(\overline{\mathbf{3}}, \mathbf{1}, 1 / 3)$ & $\tilde{d}_{R}^{c}\left(\right.$ SUSY $\left.\not R_{p}\right)$ & \\
\hline $\bar{L} d_{R}$ & $\mathcal{S}(\overline{\mathbf{3}}, \mathbf{2},-1 / 6)$ & $\tilde{Q}^{c}\left(\right.$ SUSY $\left.\not R_{p}\right)$ & 5.2 \\
\hline $\bar{L} u_{R}$ & $\mathcal{S}(\overline{\mathbf{3}}, \mathbf{2},-7 / 6)$ & & 3.6 \\
\hline$(L Q)_{t}$ & $\mathcal{S}(\overline{\mathbf{3}}, \mathbf{3}, 1 / 3)$ & & 2.5 \\
\hline \hline$(\bar{L} Q)_{s}$ & $\mathcal{V}(\overline{\mathbf{3}}, \mathbf{1},-2 / 3)$ & & \\
\hline$L d_{R}$ & $\mathcal{V}(\overline{\mathbf{3}}, \mathbf{2}, 5 / 6)$ & & \\
\hline$L u_{R}$ & $\mathcal{V}(\overline{\mathbf{3}}, \mathbf{2},-1 / 6)$ & & \\
\hline$(\bar{L} Q)_{t}$ & $\mathcal{V}(\overline{\mathbf{3}}, \mathbf{3},-2 / 3)$ & & \\
\hline
\end{tabular}

Tab. 2: Lepton-Quark Bilinears

\begin{tabular}{|c||c|}
\hline Bilinear $B$ & Couples to Boson $\mathcal{B}$ \\
\hline \hline$(\bar{Q} Q)_{s}$ & $\mathcal{V}(\mathbf{1}, \mathbf{1}, 0)$ \\
\hline$\overline{u_{R}} u_{R}$ & $\mathcal{V}(\mathbf{1}, \mathbf{1}, 0)$ \\
\hline$\overline{d_{R}} d_{R}$ & $\mathcal{V}(\mathbf{1}, \mathbf{1}, 0)$ \\
\hline$(\bar{Q} Q)_{t}$ & $\mathcal{V}(\mathbf{1}, \mathbf{3}, 0)$ \\
\hline
\end{tabular}

Tab. 3: Quark-Quark Bilinears 
In order to obtain a complete list of these operators we note that Lorentz invariance implies that any fermionic bilinear $B_{i j}$ can couple to either a scalar $(\mathcal{S})$ or a vector $(\mathcal{V})$ boson. If the two fermions of the bilinear have the same (opposite) chirality they require scalar (vector) couplings. To form a gauge invariant trilinear coupling, the boson $\mathcal{B}$ must have opposite hypercharge $Y$ and transform in the appropriate representation of $S U(2)_{L}$ and $S U(3)_{C}$. Since the SM neutrinos only appear in doublets of $S U(2)_{L}$ and since all righthanded (left-handed) charged fermions transform as $S U(2)_{L}$ singlets (doublets), it follows that any boson $\mathcal{B}$ that couples to the fermionic bilinear can only be a singlet $(s)$, a doublet $(d)$ or a triplet $(t)$ of $S U(2)_{L}$. All relevant bilinears containing only leptons are listed in Tab. 1 , and those that are built from a lepton doublet and a quark are listed in Tab. 2. In Tab. 3 we list the relevant diquark bilinears, namely, those that can couple to $\bar{L} L$. Here $Q$ and $L$ denote the left-handed SM quark and lepton doublets, and $e_{R}, u_{R}, d_{R}$ refer to the right-handed SM singlets. Some of these couplings appear in well known extensions of the standard model.

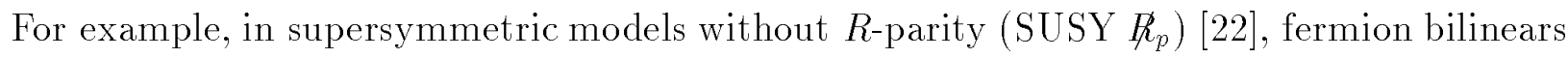
can couple to left-handed sleptons $\left(\tilde{L}^{c}\right)$, right-handed sleptons $\left(\tilde{\ell}_{R}^{c}\right)$, left-handed squarks $\left(\tilde{Q}^{c}\right)$ and right-handed down squarks $\left(\tilde{d}_{R}^{c}\right)$, as indicated in the third column of the tables. An example for a scalar triplet is the $\Delta_{L}$ in left-right symmetric models (LRSMs) [2긴.

In general any two bilinears appearing in Tab. 1-3 that couple to the same boson can be combined to a four fermion interaction with effective coupling as given in Eq. (12.1 1 ). In order to generate a non-zero $\epsilon_{\nu}^{f}$ or $\epsilon_{\nu}^{\prime f}$ in Eq. (2.2.2. a lepton doublet $L$. Clearly, four fermion operators that are the product of one bilinear and its hermitian conjugate can be constructed. If the two bilinears have the same (different) flavor structure the resulting operator will conserve (violate) lepton flavor. In addition, the $(\bar{L} L)$ bilinear can couple to $\left({\overline{e_{R}}}_{R}\right)$ or to any of the quark-quark bilinears in Tab. 3 inducing four-Fermi interactions of the form $(\bar{L} L)\left({\overline{e_{R}}}_{R}\right),(\bar{L} L)(\bar{Q} Q),(\bar{L} L)\left(\overline{u_{R}} u_{R}\right)$ and $(\bar{L} L)\left(\overline{d_{R}} d_{R}\right)$.

Note that scalar or vector fields that transform identically under the unbroken $S U(3)_{C} \otimes$ $U(1)_{E M}$ symmetry can mix. If this mixing is between a doublet and a singlet or a triplet the resulting operators violate total lepton number and are not relevant for our analysis. In the case of singlet-triplet mixing no new operators are generated. Therefore this kind of mixing does not affect our conclusions and we neglect it.

To demonstrate how $S U(2)_{L}$ related processes can be used to constrain the parameters $\epsilon_{\nu}^{f}$ or $\epsilon_{\nu}^{\prime f}$, let us consider for example the bilinear $\bar{L} f_{R}(f=e, u, d)$ that couples via a scalar doublet to its hermitian conjugate $\overline{f_{R}} L$. In terms of the component fields the effective interaction is

$$
\begin{aligned}
& \frac{\lambda_{\alpha f}^{*} \lambda_{\beta f}}{M_{1}^{2}}\left(\overline{\nu_{\alpha}} f_{R}\right)\left(\overline{f_{R}} \nu_{\beta}\right)+\frac{\lambda_{\alpha f}^{*} \lambda_{\beta f}}{M_{2}^{2}}\left(\overline{\ell_{\alpha}} f_{R}\right)\left(\overline{f_{R}} \ell_{\beta}\right) \\
= & -\frac{\lambda_{\alpha f}^{*} \lambda_{\beta f}}{2 M_{1}^{2}}\left(\overline{\nu_{\alpha}} \gamma^{\mu} \nu_{\beta}\right)\left(\overline{f_{R}} \gamma_{\mu} f_{R}\right)-\frac{\lambda_{\alpha f}^{*} \lambda_{\beta f}}{2 M_{2}^{2}}\left(\overline{\ell_{\alpha}} \gamma^{\mu} \ell_{\beta}\right)\left(\overline{f_{R}} \gamma_{\mu} f_{R}\right),
\end{aligned}
$$

where $\ell_{\alpha}=\mu_{L}, \tau_{L}$ for $\alpha=\mu, \tau . \lambda_{\alpha f}$ is the trilinear coupling of $\overline{L_{\alpha}} f_{R}$ to the scalar doublet and $M_{1,2}$ denote the masses of its $S U(2)_{L}$ components. The important point is that the 
scalar doublet exchange not only gives rise to the four-Fermi operator $\mathcal{O}_{\nu}^{f}$ in (1).2.2) (with $(V-A) \otimes(V+A)$ structure $)$, but also produces the $S U(2)_{L}$ related operator

$$
\mathcal{O}_{\ell}^{f} \equiv\left(\overline{\ell_{\alpha}} \ell_{\beta}\right)(\bar{f} f)
$$

which has the same Lorentz structure as $\mathcal{O}_{\nu}^{f}$, but where the neutrinos are replaced by their charged lepton partners. Moreover, the effective coupling of $\mathcal{O}_{\ell}^{f}$, that we denote by $G_{\alpha \beta}^{f}$, is related to $G_{\nu_{\alpha} \nu_{\beta}}^{f}$ by

$$
G_{\nu_{\alpha} \nu_{\beta}}^{f}=G_{\alpha \beta}^{f} \frac{M_{1}^{2}}{M_{2}^{2}} .
$$

Constructing all the relevant four fermion operators that are induced by the couplings between the bilinears listed in Tab. 1-3, one finds that in general $\mathcal{O}_{\ell}^{f}$ is generated together with $\mathcal{O}_{\nu}^{f^{\prime}}$. Here $f^{\prime}$ can be different from $f$ only for interactions with quarks, that is in some cases $\mathcal{O}_{\ell}^{u}\left(\mathcal{O}_{\ell}^{d}\right)$ is generated together with $\mathcal{O}_{\nu}^{d}\left(\mathcal{O}_{\nu}^{u}\right)$. The leptonic operator $\mathcal{O}_{\ell}^{e}$ is always generated together with $\mathcal{O}_{\nu}^{e}$ unless the interaction is mediated by an intermediate scalar $S U(2)_{L}$ singlet that couples to

$$
\left(L_{\alpha} L_{e}\right)_{s}=\frac{1}{\sqrt{2}}\left(\overline{\nu_{\alpha}^{c}} e_{L}-\overline{\ell_{\alpha}^{c}} \nu_{e}\right)
$$

where $\ell_{\alpha}=\mu_{L}, \tau_{L}$ for $\alpha=\mu, \tau$, with the elementary coupling $\lambda_{\alpha e}$. The coupling of $\left(L_{\alpha} L_{e}\right)_{s}$ to $\left(L_{\beta} L_{e}\right)_{s}^{\dagger}$ that is mediated by a scalar singlet of mass $M$ yields the effective interactions

$$
\begin{aligned}
& \frac{\lambda_{\beta e}^{*} \lambda_{\alpha e}}{M^{2}}\left[\left(\overline{e_{L}} \nu_{\beta}^{c}\right)\left(\overline{\nu_{\alpha}^{c}} e_{L}\right)-\left(\overline{e_{L}} \nu_{\beta}^{c}\right)\left(\overline{\ell_{\alpha}^{c}} \nu_{e}\right)+\left(\overline{\nu_{e}} \ell_{\beta}^{c}\right)\left(\overline{\ell_{\alpha}^{c}} \nu_{e}\right)-\left(\overline{\nu_{e}} \ell_{\beta}^{c}\right)\left(\overline{\nu_{\alpha}^{c}} e_{L}\right)\right]= \\
& \frac{\lambda_{\beta e}^{*} \lambda_{\alpha e}}{2 M^{2}}\left[\left(\overline{e_{L}} \gamma^{\mu} e_{L}\right)\left(\overline{\nu_{\beta}} \gamma_{\mu} \nu_{\alpha}\right)-\left(\overline{e_{L}} \gamma^{\mu} \nu_{e}\right)\left(\overline{\nu_{\beta}} \gamma_{\mu} \ell_{\alpha}\right)+\left(\overline{\nu_{e}} \gamma^{\mu} \nu_{e}\right)\left(\overline{\ell_{\beta}} \gamma_{\mu} \ell_{\alpha}\right)-\left(\overline{\nu_{e}} \gamma^{\mu} e_{L}\right)\left(\overline{\ell_{\beta}} \gamma_{\mu} \nu_{\alpha}\right)\right],
\end{aligned}
$$

where we used a Fierz transformation and the identity $\overline{A^{c}} \gamma^{\mu} B^{c}=-\bar{B} \gamma^{\mu} A$ to obtain (2).8. One can see that in this case $\mathcal{O}_{\nu}^{e}$ is generated together with three more operators that have the same effective coupling (up to a sign). However, unlike for the case of intermediate doublets or triplets, all these operators involve two charged leptons and two neutrinos.

\section{B. Experimental constraints}

There is no experimental evidence for any non-vanishing $G_{\mu \tau}^{f}$. Therefore, whenever $\mathcal{O}_{\ell}^{f}$ is generated together with $\mathcal{O}_{\nu}^{f}$, one can use the upper bounds on $G_{\mu \tau}^{f}$ to derive constraints

on $G_{\nu_{\mu} \nu_{\tau}}^{f}$. The most stringent constraint on $G_{\mu \tau}^{e}$ is due to the upper bound on $\tau^{-} \rightarrow$

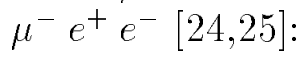

$$
\operatorname{BR}\left(\tau^{-} \rightarrow \mu^{-} e^{+} e^{-}\right)<1.7 \times 10^{-6}
$$


Normalizing the above bound to the measured rate of the related lepton flavor conserving

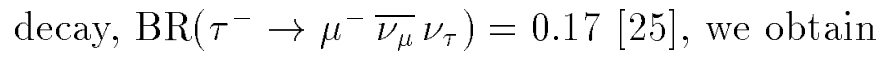

$$
G_{\mu \tau}^{e}<3.1 \times 10^{-3} G_{F}
$$

To constrain $G_{\mu \tau}^{q}$ we may use the upper bounds on various semi-hadronic tau decays that violate lepton flavor [12 $\left[\begin{array}{l}2 \\ 1\end{array} ; 25\right]$ :

$$
\begin{aligned}
\operatorname{BR}\left(\tau^{-} \rightarrow \mu^{-} \pi^{0}\right) & <4.0 \times 10^{-6}, \\
\operatorname{BR}\left(\tau^{-} \rightarrow \mu^{-} \rho^{0}\right) & <6.3 \times 10^{-6} \\
\operatorname{BR}\left(\tau^{-} \rightarrow \mu^{-} \eta\right) & <9.6 \times 10^{-6} \\
\operatorname{BR}\left(\tau^{-} \rightarrow \mu^{-} \pi^{+} \pi^{-}\right) & <8.2 \times 10^{-6}
\end{aligned}
$$

Let us first consider the tau decays into $\pi^{0}$ and $\rho^{0}$. Since these mesons belong to an isospin

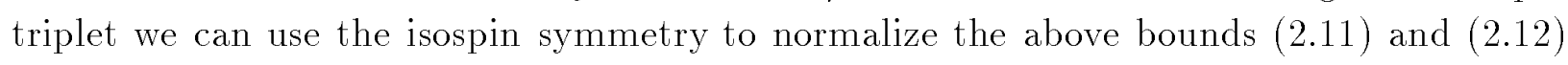
by the measured rates of related lepton flavor conserving decays. Using $\operatorname{BR}\left(\tau^{-} \rightarrow \nu_{\tau} \pi^{-}\right)=$

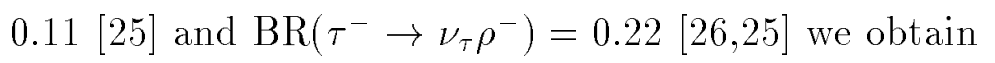

$$
G_{\mu \tau}^{q}(\pi)<8.5 \times 10^{-3} G_{F} \quad \text { and } \quad G_{\mu \tau}^{q}(\rho)<7.5 \times 10^{-3} G_{F}
$$

Since the $\pi(\rho)$ is a pseudoscalar (vector) meson its decay probes the axial-vector (vector) part of the quark current.

In general, any semi-hadronic operator $\mathcal{O}_{\ell}^{q}$ can be decomposed into an $I=0$ and an $I=1$ isospin component. Only the effective coupling of the latter can be constrained by the upper bounds on the decays into final states with isovector mesons, like the $\pi$ and the $\rho$. If the resulting operator is dominated by the $I=0$ component, the bounds in (2.15) do not hold. But in this case we can use the upper bound on $\operatorname{BR}\left(\tau^{-} \rightarrow \mu^{-} \eta\right)$ in $(2,13)$. Since the $\eta$ is an isosinglet, isospin symmetry is of no use for the normalization. However, we can estimate the proper normalization using the relation between the $\eta$ and $\pi$ hadronic matrix elements,

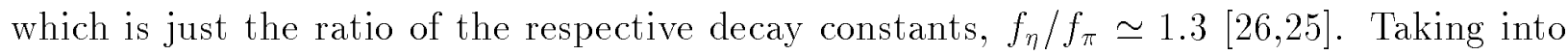
account the phase space effects, we obtain from ( $\left(12 . \overline{2} 3_{1}\right)$ that

$$
G_{\mu \tau}^{q}(\eta)<1.2 \times 10^{-2} G_{F} .
$$

Since the $\eta$ is a pseudoscalar meson its decay probes the axial-vector part of the $I=0$ component of the quark current, while the neutrino propagation is only affected by the vector part. As we have already mentioned, for any single chiral New Physics contribution

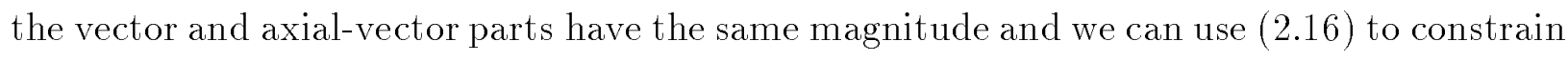
the isosinglet component of $\mathcal{O}_{\ell}^{q}$. In case there are several contributions, whose axial-vector parts cancel each other (a vector singlet $\mathcal{V}(\mathbf{1}, \mathbf{1}, 0)$ that couples to all the diquark singlets of Tab. 3 with the same strength would lead to such a scenario), the $I=0$ component could still be constrained by the upper bound on $\operatorname{BR}\left(\tau^{-} \rightarrow \mu^{-} \pi^{+} \pi^{-}\right)$in (2.14is $)$. While the calculation of the rate is uncertain due to our ignorance of the spectra and the decay constants of the 
isosinglet scalar resonances, we expect that the normalization will be similar to that of the $\pi, \rho$ and $\eta$ discussed before. Finally we note that the decay $\tau^{-} \rightarrow \mu^{-} \omega$ would be ideal to constrain the $I=0$ vector part, but at present no upper bound on its rate is available.

While one can always fine-tune some parameters in order to avoid our bounds, our basic assumption is that this is not the case. Thus from ( $\left(5.10^{\prime}\right),\left(\begin{array}{c}2.15 \\ 2\end{array}\right)$ and $\left(2.1 \overline{6}^{\prime}\right)$ we conclude that the effective coupling $G_{\mu \tau}^{f}$ could be at most at the one-percent level.

We still have to discuss the case of the intermediate scalar singlet that couples to $(L L)_{s}$ inducing the effective interactions in (2.8. Since $\mathcal{O}_{\nu}^{e}$ is not generated together with $\mathcal{O}_{\ell}^{e}$ but only with operators that involve two charged leptons and two neutrinos, the decay $\tau^{-} \rightarrow \mu^{-} e^{+} e^{-}$is of no use to constrain the effective couplings. However, since the effective operators in (2. $\left.\overline{8}_{1}^{\prime}\right)$ contain only left-handed fermions rather strong bounds can be derived on the flavor diagonal terms using lepton universality. The reason is that the corresponding interactions are identical to the SM ones and have to be added coherently.

Setting $\alpha=\beta=\mu, \tau$ the last term in (2. $\left.\overline{8}_{1}^{\prime}\right)$ induces additional contributions to $\ell_{\alpha} \rightarrow$ $e_{L} \nu_{\alpha} \overline{\nu_{e}}$ with the effective coupling

$$
G_{\nu_{\alpha} \nu_{\alpha}}^{e}=\frac{\left|\lambda_{\alpha e}\right|^{2}}{4 \sqrt{2} M^{2}}
$$

These new contributions violate lepton universality and lead to a deviation of the parameter

$$
R_{\tau / \mu} \equiv \sqrt{\frac{1}{N} \frac{\Gamma\left(\tau^{-} \rightarrow e^{-} \nu_{\tau} \overline{\nu_{e}}\right)}{\Gamma\left(\mu^{-} \rightarrow e^{-} \nu_{\mu} \overline{\nu_{e}}\right)}} \approx 1+\frac{G_{\nu_{\tau} \nu_{\tau}}^{e}-G_{\nu_{\mu} \nu_{\mu}}^{e}}{G_{F}}
$$

from unity. Here $N$ denotes a normalization factor, which is just the ratio of the above two rates in the $\mathrm{SM}$ such that $R_{\tau / \mu}=1$ if $G_{\nu_{\alpha} \nu_{\alpha}}^{e}=0$. In the approximation we assume that $G_{\nu_{\alpha} \nu_{\alpha}}^{e} \ll G_{F}(\alpha=\mu, \tau)$. From the most recent experimental data $[27250.51$ it follows that

$$
R_{\tau / \mu}=1.0008 \pm 0.0030
$$

implying that

$$
\epsilon_{\nu}^{\prime e}=\frac{G_{\nu_{\tau} \nu_{\tau}}^{e}-G_{\nu_{\mu} \nu_{\mu}}^{e}}{G_{F}}<3.8 \times 10^{-3}
$$

Here we used that $\mathcal{O}_{\nu}^{e}$ has the same effective coupling as the related operator that induces the new contribution to $\ell_{\alpha} \rightarrow e_{L} \nu_{\alpha} \overline{\nu_{e}}$.

Finally we remark that we can use lepton universality violation not only to constrain the interactions induced by an intermediate singlet, but our argument holds also whenever an $S U(2)_{L}$ related operator induces additional contributions to the SM weak interactions. The bounds on lepton universality violation in semi-hadronic processes [2] $\left.\overline{2}_{1}^{1}\right]$ are of similar

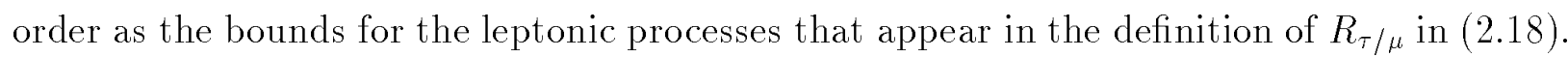
Consequently analogous arguments as those leading to the upper bound on $\epsilon_{\nu}^{\prime}$ in $\left(\overline{2} \cdot \overline{2} 0^{\prime}\right)$ can be used to constrain $\epsilon_{\nu}^{\prime q}$. Since all involved fermions have to be left-handed this only applies for intermediate singlets or triplets of $S U(2)_{L}$. For the triplets the effective couplings of the relevant operators may differ due to $S U(2)_{L}$ breaking effects, which we will study next. 


\section{Constraining $S U(2)_{L}$ breaking effects}

If $S U(2)_{L}$ breaking effects are negligible then $G_{\nu_{\alpha} \nu_{\beta}}^{f}$ is equal to $G_{\alpha \beta}^{f}$. Comparing the experimental bounds $\left(\begin{array}{c}1 \\ 2\end{array} \overline{0}^{\prime}\right),\left(\begin{array}{c}2 \\ 2\end{array}\right.$ symmetric limit the new neutrino interactions that we considered cannot have a significant contribution to the AN anomaly.

The excellent agreement between the SM predictions and the electroweak precision data implies that $S U(2)_{L}$ breaking effects cannot be large. To show that they cannot sufficiently weaken the upper bounds on $\epsilon_{\nu}^{f}$ and $\epsilon_{\nu}^{f}$ to be consistent with (ㄴ.1ㄱ. that in general the ratio of the couplings, $G_{\nu_{\alpha} \nu_{\beta}}^{f} / G_{\alpha \beta}^{f}$, is given by ratio $M_{1}^{2} / M_{2}^{2}$. Here $M_{1}$ and $M_{2}$ are the masses of the particles belonging to the $S U(2)_{L}$ multiplet that mediate the processes described by $G_{\alpha \beta}^{f}$ and $G_{\nu_{\alpha} \nu_{\beta}}^{f}$, respectively. If $M_{1} \neq M_{2}$ this multiplet will

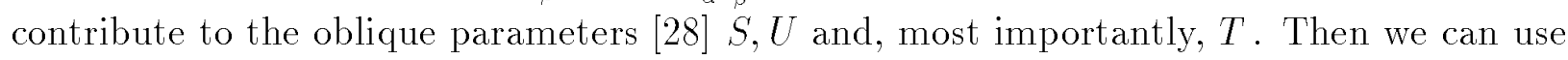
a fit to the precision data to determine the maximally allowed ratio $\left(M_{1} / M_{2}\right)_{\max }^{2}$.

We use the program GAPP by J. Erler [돔] to calculate the SM predictions. For the latest precision data from the $Z$-pole $\left[\begin{array}{l}3 \\ 3\end{array}\right]$

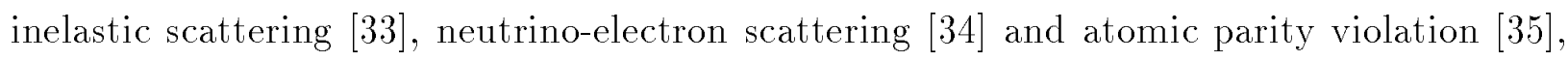
we obtain the best fit values of the oblique parameters:

$$
S=-0.07 \pm 0.11, \quad T=-0.10 \pm 0.14, \quad U=0.11 \pm 0.15
$$

We calculate the contributions to $S, T$, and $U$ from the various scalar representations in Tab. 1 and Tab. 2 and determine the best fit to the data at each value of the mass splitting. The best fit Higgs mass $M_{H}$ varies with the splitting, and we limit the Higgs mass to the range $95 \mathrm{GeV}<M_{H}<1 \mathrm{TeV}$. Constructing a $\chi^{2}$ function we determine the upper bound on the mass splitting between the different members of a multiplet at a given confidence level (CL). The individual $90 \% \mathrm{CL}$ bounds on $\left(M_{1} / M_{2}\right)^{2}$ are given in the last column of Tab. 1 and Tab. 2. (Note that the limit on $\left(M_{1} / M_{2}\right)^{2}$ is stronger if the lightest mass is heavier. From the $Z$-width measurement, the lightest mass must be heavier than $M_{Z} / 2$, and the bounds presented in the tables are derived for the case where the lighter mass is $50 \mathrm{GeV}$.)

We did not calculate the bounds for the vector multiplets. Since vector bosons give in general larger contributions, we expect the bounds in the vector cases to be as good or better than the corresponding bounds for the scalar multiplets. Thus, for the vector multiplets a rather conservative upper bound is $\left(M_{1} / M_{2}\right)^{2}<7$.

Hence, even the maximal possible $S U(2)_{L}$ breaking effects could weaken the bounds we derived only by a factor of a few and $\epsilon_{\nu}^{f}, \epsilon^{\prime f}{ }_{\nu}$ cannot exceed the few-percent level. We learn that also the flavor changing neutrino scattering $\nu_{\mu} f \rightarrow \nu_{\tau} f$ induced by heavy bosons, that are doublets or triplets of $S U(2)_{L}$, cannot significantly contribute to the AN anomaly. 


\section{Z-INDUCED FLAVOR CHANGING NEUTRAL CURRENTS}

In the previous section we considered models where the only modification to the neutrino sector is due to new interactions mediated by heavy bosons. In this section we study the opposite scenario, where new fermions are added, but no extra bosons beyond the SM ones are needed. As an example we consider Z-induced FCNCs that arise when introducing a heavy sterile neutrino. Such SM gauge singlets, which appear in many extensions of the standard model, are frequently employed to explain the smallness of the neutrino masses

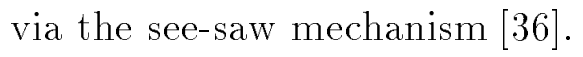

The basic idea for $Z$-induced FCNCs is that if a neutrino interaction eigenstate is a linear combination of light and heavy mass eigenstates then the effective low-energy interaction eigenstates, that consist only of light mass eigenstates, are not orthogonal to each other [3] Thus the couplings to the $Z$-boson (and in fact also to the $W$-boson) have to be modified slightly, implying that also the effective Hamiltonian that describes the neutrino propagation in matter has to be changed.

In Ref. [i] 18 a general discussion of $Z$-induced FCNCs and their impact on neutrino oscillations has been presented. In the context of the AN problem we illustrate the mechanism by considering a simple framework where besides the SM neutrinos $\nu_{\mu}$ and $\nu_{\tau}$ there is only one new gauge singlet $\nu_{S}$. (For simplicity we assume that the $\nu_{e}$ does not play an important role here.) These interaction eigenstates are connected to the mass eigenstates by a unitary transformation

$$
\left(\begin{array}{c}
\nu_{\mu} \\
\nu_{\tau} \\
\nu_{S}
\end{array}\right)=\left(\begin{array}{ccc}
U_{\mu 1} & U_{\mu 2} & U_{\mu h} \\
U_{\tau 1} & U_{\tau 2} & U_{\tau h} \\
U_{S 1} & U_{S 2} & U_{S h}
\end{array}\right)\left(\begin{array}{c}
\nu_{1} \\
\nu_{2} \\
\nu_{h}
\end{array}\right),
$$

where $\nu_{1}$ and $\nu_{2}$ denote the light and $\nu_{h}$ the heavy mass eigenstates. The neutrinos that are produced in low-energy charged-current interactions together with charged leptons $\mu$ and $\tau$ are

$$
\left(\begin{array}{c}
\nu_{\mu}^{P} \\
\nu_{\tau}^{P}
\end{array}\right)=\left(\begin{array}{ll}
U_{\mu 1} & U_{\mu 2} \\
U_{\tau 1} & U_{\tau 2}
\end{array}\right)\left(\begin{array}{l}
\nu_{1} \\
\nu_{2}
\end{array}\right),
$$

i.e. we have projected $\nu_{\mu}$ and $\nu_{\tau}$ onto the $\nu_{1}-\nu_{2}$ plane. Since the mixing matrix appearing in $\left(\begin{array}{l}3.2 \\ 1\end{array}\right)$ is only a submatrix of the unitary matrix in $\left(\underline{b} . \overline{1}_{1}^{\prime}\right), \nu_{\mu}^{P}$ and $\nu_{\tau}^{P}$ are not orthogonal to each other

$$
\left\langle\nu_{\mu}^{P} \mid \nu_{\tau}^{P}\right\rangle=U_{\mu 1}^{*} U_{\tau 1}+U_{\mu 2}^{*} U_{\tau 2}=-U_{\mu h}^{*} U_{\tau h}
$$

and also not properly normalized. Consequently these states do not provide a proper basis for the neutrino oscillation formalism.

The description of neutrino oscillation in the presence of heavy gauge singlets has been worked out in Ref. [표묘. The main result is that the effective non-unitary mixing induces a flavor off-diagonal contribution in the matter-induced neutrino potential $V_{F C N C}$. The effect 
is proportional to the neutron density and its size is characterized by the ratio between $V_{F C N C}$ and the standard (flavor diagonal) neutral current (NC) potential $V_{N C}$ :

$$
\epsilon_{\nu}^{Z}=\frac{V_{F C N C}}{V_{N C}} \simeq\left|U_{\mu h}^{*} U_{\tau h}\right|
$$

The approximation refers to the simple example we discussed previously. It reveals that the effect is in general small, since it is proportional to the components of the known neutrinos along the heavy mass-eigenstates, which cannot be large. The $Z$-induced FCNCs cannot be constrained by the $S U(2)_{L}$-related charged lepton decay, that we used before in the context of FCNIs due to heavy particle exchange, but one can obtain a stringent constraint on $\epsilon_{\nu}^{Z}$ from a global fit using lepton universality, CKM unitarity, and the measured $Z$ invisible decay width [3]

$$
\left|U_{\mu h}\right|^{2}<0.0096 \quad \text { and } \quad\left|U_{\tau h}\right|^{2}<0.016
$$

We conclude that the parameter $\epsilon_{\nu}^{Z}$ in $(\overline{3} . \overline{4})$ cannot exceed the few-percent level. Therefore from ( $\left(1 .{ }_{1}\right)$ it follows that $Z$-induced FCNC effects are too small to be relevant for the AN problem.

\section{CONCLUSIONS}

Extensions of the standard model in general do not conserve lepton flavor and therefore provide an alternative mechanism for neutrino flavor conversion that may show up in neutrino oscillation experiments. While such a scenario where flavor changing neutrino interactions (FCNIs) explain one of the three neutrino anomalies is a priori well motivated, one has to check carefully whether these solutions are phenomenologically viable. In [i] $\overline{3}$ ] it was shown that FCNIs cannot be large enough to explain the LSND anomalies. In this paper we argue that it is also very unlikely that the AN anomaly is due to FCNIs. Both analysis rely on three facts:

- The neutrino flavor changing four fermion operator that is induced by the exchange of a heavy boson is related by an $S U(2)_{L}$ rotation to other operators that violate lepton flavor.

- The strength of these related operators is severely constrained by the upper bounds on lepton number violating processes.

- High precision measurements imply that the violation of the $S U(2)_{L}$ symmetry is not large for new physics at or above the weak scale. Consequently the upper bounds on the operators that induce FCNIs are of the same order as those of the $S U(2)_{L}$ related operators. 
The first point follows immediately from the fact that the SM neutrinos appear in $S U(2)_{L}$ doublets. Using the upper bounds on $\tau^{-} \rightarrow \mu^{-} M\left(M=\pi^{0}, \rho^{0}, \eta\right)$ and $\tau^{-} \rightarrow \mu^{-} e^{+} e^{-}$ we constrain, in a model-independent way, the strength of the flavor changing neutrino scattering reaction $\nu_{\mu} f \rightarrow \nu_{\tau} f$ to be at most at the one-percent level (compared with $G_{F}$ ). For the unique case of an intermediate scalar singlet we derive a severe constraint on the nonuniversal flavor diagonal neutrino interactions using the upper bound on lepton universality violation.

The constraints we obtained for the parameters that describe the new neutrino interactions are not consistent with the values that are required to explain the $\mathrm{AN}$ anomaly in terms of FCNIs. Thus we conclude that such a solution is ruled out. One could evade our bounds by fine-tuning several new physics contributions, but we do not consider such a scenario as very attractive. Nevertheless, we would like to stress that ultimately any alternative explanation [3.9. of the AN anomaly should be tested by the experimental data itself. For the time being "standard" neutrino oscillations with massive neutrinos remain the most plausible and elegant solution.

\section{ACKNOWLEDGMENTS}

We thank K.S. Babu, J. Erler, Y. Nir and S. Pakvasa for helpful discussions. Y.G. is supported by the U.S. Department of Energy under contract DE-AC03-76SF00515. D.M.P. is supported under Department of Energy contract DE-AC02-98CH10886. 


\section{REFERENCES}

[1] H.S. Hirata et al., Phys. Lett. B 280, 146 (1992);

Y. Fukuda et al., Phys. Lett. B 335, 237 (1994).

[2] R. Becker-Szendy et al., Phys. Rev. D 46, 3720 (1992); Phys. Rev. Lett. 69, 1010 (1992).

[3] W.W.M. Allison et al., Phys. Lett. B 391, 49 (1997); Phys. Lett. B 449, 137 (1999).

[4] Y. Fukuda et al., Phys. Lett. B 433, 9 (1998); Phys. Lett. B 436, 33 (1998).

[5] E. Ma and P. Roy, Phys. Rev. Lett. 80, 4637 (1998) [hep-ph

[6] G. Brooijmans, h̆ep=ph

[7] M.C. Gonzalez-Garcia et al., Phys. Rev. Lett. 82, 3202 (1999) [hep-ph/9809531'.

[8] N. Fornengo, M.C. Gonzalez-Garcia, H. Nunokawa and J.W.F. Valle, hiep-ph/9906

[9] For recent analysis, see e.g.:

M.C. Gonzalez-Garcia, et al., Phys. Rev. D 58, 033004 (1998);

M.C. Gonzalez-Garcia, H. Nunokawa, O.L.G. Peres and J.W.F. Valle,

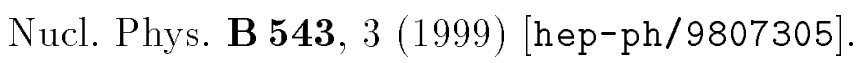

[10] J.N. Bahcall and P.I. Krastev, Phys. Rev. D 53, 4211 (1996);

N. Hata and P. Langacker, Phys. Rev. D 56, 6107 (1997);

J.N. Bahcall, P.I. Krastev and A.Yu. Smirnov, Phys. Rev. D 58, 096016 (1998).

[11] LSND collab.: C. Athanassopoulos et al., Phys. Rev. Lett. 77, 3082 (1996); Phys. Rev. Lett. 81, 1774 (1998).

[12] See for example:

D.O. Caldwell and R.N. Mohapatra, Phys. Rev. D 48, 3259 (1993); ibid. 50, 3477 (1994);

J.T. Peltoniemi and J.W.F. Valle, Nucl. Phys. B 406, 409 (1993);

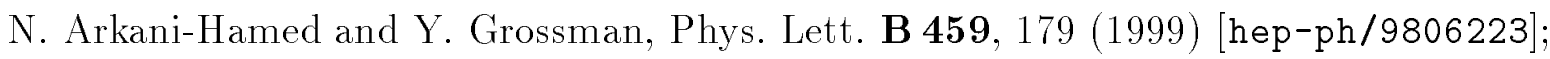

G. Dvali and Y. Nir, JHEP 9810, 014 (1998) [hep-ph $\overline{9} \overline{9} \overline{8} \overline{1} 0 \overline{2} \overline{5} \overline{7}$, and references therein.

[13] S. Bergmann and Y. Grossman, Phys. Rev. D 59, 093005 (1999) 觔

[14] E. Roulet, Phys. Rev. D 44, 935 (1991);

M.M. Guzzo, M. Masiero and S.T. Petcov, Phys. Lett. B 260, 154 (1991);

S. Degl'Innocenti and B. Ricci, Mod. Phys. Lett. A 8, 471 (1993);

G. L. Fogli and E. Lisi, Astroparticle Phys. 2, 91 (1994);

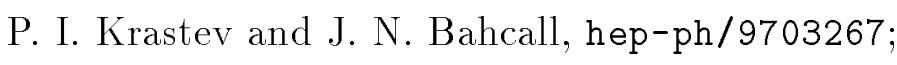

S. Bergmann, Nucl. Phys. B 515, 363 (1998) [hำ-ph/9̄7ō

[15] V. Barger, R. J. N. Phillips and K. Whisnant, Phys. Rev. D 44, 1629 (1991). 
[16] S. Bergmann, Y. Grossman and E. Nardi, heph Phys. Rev. D.

[17] P. Langacker and D. London, Phys. Rev. D 38, 907 (1988);

H. Nunokawa, Y.-Z. Qian, A. Rossi and J. W. F. Valle, Phys. Rev. D 54, 4356 (1996)

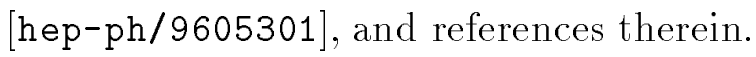

[18] S. Bergmann and A. Kagan, Nucl. Phys. B 538, 368 (1999) [hep-ph/9803305].

[19] P. Lipari and M. Lusignoli, Phys. Rev. D 60, 013003 (1999) [ihep-ph

[20] G.L. Fogli, E. Lisi, A. Marrone and G. Scioscia, Phys. Rev. D 60, 053006 (1999) [ihep-ph/9904248̄in.

[21] M.M. Guzzo, et al. , ihep-ph/9908308'.

[22] C. S. Aulakh and N. R. Mohapatra, Phys. Lett. B 119, 136 (1983);

F. Zwirner, Phys. Lett. B 132, 103 (1983);

L. J. Hall and M. Suzuki, Nucl. Phys. B 231, 419 (1984);

J. Ellis et al., Phys. Lett. B 150, 14 (1985);

G. G. Ross and J. W. F. Valle, Phys. Lett. B 151, 375 (1985);

R. Barbieri and A. Masiero, Phys. Lett. B 267, 679 (1986).

[23] J. C. Pati and A. Salam, Phys. Rev. D 10, 275 (1974);

R. N. Mohapatra and J. C. Pati, Phys. Rev. D 11, 566; ibid 2558 (1975);

R. N. Mohapatra and G. Senjanović, Phys. Rev. D 12, 1502 (1975);

See also Ref. [i]

[24] CLEO collab.: W. Bliss et al., Phys. Rev. D 57, 5903 (1998) [ihep-ex/9712010in.

[25] Particle Data Group: C. Caso et al., Eur. Phys. J. C 3, 1 (1998); see also:

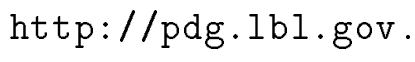

[26] See, e.g., J.F. Donoghue, E. Golowich and B.R. Holstein, Dynamics of the Standard Model, Cambridge University Press, 1992.

[27] A. Pich, Review talk at Lepton Photon 99, Stanford University, August, 1999. See also: hep-ph/971129, hep-ph/9802257.

[28] M.E. Peskin and T. Takeuchi, Phys. Rev. Lett. 65, 964 (1990) and Phys. Rev. D 46, 381 (1992).

[29] J. Erler, Phys. Rev. D 59, 054008 (1999); see also:

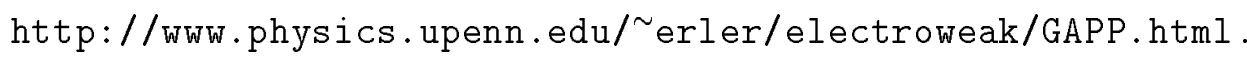


[30] J. Mnich and G. Quast, presented at the International Europhysics Conference on High Energy Physics, Tampere (1999);

The LEP Collaborations ALEPH, DELPHI, L3, OPAL, the LEP Electroweak Working Group and the SLD Heavy Flavour and Electroweak Groups: D. Abbaneo et al., CERN-EP/99-15;

R. Clare, private communication to J. Erler;

The LEP/SLD Heavy Flavour Working Group: D. Abbaneo et al., LEPHF/99-01;

SLD: K. Abe et al., Phys. Rev. Lett. 78, 2075 (1997);

SLD: J.E. Brau et al., presented at the International Europhysics Conference on High Energy Physics, Tampere (1999);

SLD: K. Abe et al., submitted to the International Europhysics Conference on High Energy Physics, Tampere (1999);

SLD: K. Abe et al., Phys. Rev. Lett. 79, 804 (1997);

SLD: K. Abe et al., Phys. Rev. Lett. 78, 17 (1997);

DELPHI: P. Abreu et al., Z. Phys. C 67, 1 (1995);

OPAL: K. Ackerstaff et al., submitted to the XXIXth Conference on High Energy Physics, Vancouver (1998).

[31] CDF: F. Abe et al., Phys. Rev. Lett. 75, 11 (1995);

CDF: F. Abe et al., Phys. Rev. D 52, 4784 (1995);

CDF: W. Carithers et al., presented at the International Europhysics Conference on High Energy Physics, Tampere (1999);

D0: B. Abbott et al., Phys. Rev. D 58, 092003 (1998);

D0: B. Abbott et al., Phys. Rev. Lett. 80, 3008 (1998);

D0: B. Abbott et al., submitted to the International Europhysics Conference on High Energy Physics, Tampere (1999);

UA2: S. Alitti et al., Phys. Lett. B 276, 354 (1992).

[32] CDF: F. Abe et al., Phys. Rev. Lett. 82, 271 (1999);

D0: B. Abbott et al., Phys. Rev. D 58, 052001 (1998).

[33] NuTeV collab.: K.S. McFarland et al., hiep-ex/9806013', submitted to the Proceedings of the XXXIIIrd Rencontres de Moriond: Electroweak Interactions and Unified Theories, Les Arcs, France, March 1998;

CCFR collab.: K.S. McFarland et al., Eur. Phys. J. C 1, , 509 (1998);

CDHS collab.: H. Abramowicz et al., Z. Phys. C 28, 51 (1985), and A. Blondel et al., Z. Phys. C 45, 361 (1990);

CHARM collab.: V. Allaby et al., Z. Phys. C 36, 611 (1987).

[34] CHARM II collab.: P. Vilain et al., Phys. Lett. B 335, 246 (1994). 
[35] C.S. Wood et al., Science 2, 7 5, 1759 (1997); S.C. Bennett and C.E. Wieman, Phys. Rev. Lett. 82, 2484 (1999); N.H. Edwards, S.J. Phipp, P.E.G. Baird, and S. Nakayama, Phys. Rev. Lett. 74, 2654 (1995); P.A. Vetter, et al., Phys. Rev. Lett. 74, 2658 (1995).

[36] T. Yanagida, Proceedings of the Workshop on Unified Theory and Baryon Number in the Universe, (KEK, Japan, 1979);

M. Gell-Mann, R. Slansky and P. Ramond, Supergravity, (North Holland 1979) 346.

[37] J. Schechter and J. W. F. Valle Phys. Rev. D 22, 2227 (1980);

P. Langacker and D. London, Phys. Rev. D 38, 907 (1988), and references therein.

[38] E. Nardi, E. Roulet and D. Tommasini, Phys. Lett. B 344, 225 (1995);

C. P. Burgess et al., Phys. Rev. D 49, 6115 (1994);

P. Langacker and D. London, Phys. Rev. D 38, 886 (1988).

[39] S. Pakvasa, ihep ph 